\title{
Synaptic Spinules Attendant on Post-tetanic Potentiation in Cat Sympathetic Ganglion
}

\author{
By Tomoko Kadota, ${ }^{*)+}$ ) Muneaki Mizote, ${ }^{* *)}$ and Ken Kadota***) \\ (Communicated by Setsuro EBASHI, M. J. A., March 12, 1996)
}

\begin{abstract}
A synaptic spinule formed on the postsynaptic membrane in the cat superior cervical ganglion (SCG) in vivo was electron microscopically studied. When the preganglionic nerve fiber was tetanically stimulated at $10 \mathrm{~Hz}$ for more than 10 seconds, a post-tetanic potentiation (PTP) was electrophysiologically detected and maintained up to $30 \mathrm{~min}$. Concurrently with PTP, a small process, termed as synaptic spinule, was generated as a small projection standing out from the postsynaptic dendritic membrane with a straightly extended contour. The spinule produced a break at about a middle point of the postsynaptic density (PSD) and divided it into two parts forming a perforated synapse. Then, the spinule increased in size, invaginated into the cytoplasm of the nerve terminal, and subdivided the presynaptic terminal bag into two compartments. Each of the compartments retained a morphology of an active synaptic site with a cluster of synaptic vesicles associated with a presynaptic active zone standing opposite to the one part of the subdivided PSD. The longer the tetanic stimulation in period, the more the synapses with the spinule increased in number. In addition to this, a few spinules (multiple type of spinule) were often formed after the stimulation of $1 \mathrm{~min}$ and more than two compartments were generated in a single nerve ending. The present observations have suggested that the synaptic spinule is a morphological representation of the "synaptic plasticity" which is electrophysiologically displayed as the PTP in cat SCG in vivo.
\end{abstract}

Key words: Synaptic spinule; postsynaptic dendritic membrane; post-tetanic potentiation; synaptic plasticity; perforated synapse; sympathetic ganglion.

Introduction. The spinule at the synapse was first described in the rat hippocampus as a finger-like extension of the postsynaptic elements of a dendritic spine projecting into the presynaptic terminal at the asymmetrical synapse. ${ }^{1)}$ Similar structures have been observed in the nerve cells of the rat telencephalon. ${ }^{2)}$ Despite a wide occurrence of spinules in the central nervous system (CNS), their roles in synaptic transmission have remained elusive for a long period. Recently, Geinisman ${ }^{3), 4)}$ has found that the spinule formation is induced by the long term potentiation (LTP) or kindling in the hippocampus and that the perforated synapse is often attendant on the spinule formation. Moreover, Wagner \& Djamgoz ${ }^{5)}$ have found

*) Department of Anatomy, Chiba University Medical School, Chiba 260, Japan.

**) Department of Engineering Information, University of Teikyo Heisei, Chiba 290-02, Japan.

***) Medical Department, Chiba Center of Social Welfare for Mentally Retarded, Chiba 299-02, Japan.

t) Correspondence to: T. Kadota. the spinule formation in the fish retina during light adaptation and suggested that the spinule may relate to the retinal synaptic plasticity. Edwards ${ }^{(6)}$ has implicated that LTP might serve as a phenomenon to combine anatomical and electrophysiological changes. He has speculated that the perforated synapse resulting from the spinule formation might induce the increase of active synaptic sites in number, affect the change of synaptic efficacy, and relate to the induction of LTP.

The previous report from this laboratory has shown that a tetanic stimulation at $10 \mathrm{~Hz}$ induces a post-tetanic potentiation (PTP) correlating to the increase of the synaptic vesicle number in the cat SCG. ${ }^{7)}$ Attempting to supplement this presynaptic plasticity following the tetani, the authors have focused the attention on clarifying possible postsynaptic morphological changes attendant on PTP.

Materials and methods. Cats of either sex, weighing 2-4 kg, were anesthetized with sodium pentobarbital, i.p. at a dose $35 \mathrm{mg} / \mathrm{kg}$. Both sides of 
SCG were prepared and the preganglionic nerve fibers were electrically stimulated supramaximally at $10 \mathrm{~Hz}$ with a square pulse of $0.5 \mathrm{msec}$ duration for predetermined period ( $1 \mathrm{sec}-30 \mathrm{~min})$ as previously described. $^{7)}$ The postganglionic compound action potentials (PGP) were recorded. The ganglia were fixed by local perfusion via the lingual artery for electron microscopy. ${ }^{8)}$

Results. The preganglionic sympathetic trunk was stimulated at $10 \mathrm{~Hz}$ during various periods from 1 sec to $30 \mathrm{~min}$. The amplitude of PGP was compared with that in response to a single shock at rest immediately before starting the tetanic stimulation. The PGP showed an increase in amplitude by ca $30 \%$ of the control after $10 \mathrm{sec}$ of stimulation. The authors referred to this increase of PGP as a post-tetanic potentiation (PTP), which was kept during whole stimulation period of $30 \mathrm{~min}$.

The preganglionic nerve fiber was usually observed to form a typical axodendritic asymmetrical synapse on the dendrite of the ganglion cells (Fig. 1a). Under the resting condition the synaptic complex consisting of the pre- and postsynaptic membranes was usually straight and smooth in contour. The active zone (presynaptic dense projection) and postsynaptic density (PSD) continuously lined the cytoplasmic sides of both synaptic membranes. The perforated synapse, a synapse with a break of PSD nearly at the middle point, was found very rarely, fewer than $3 \%$ of all synapses, in the unstimulated control material.
When the preganglionic nerve fibers were tetanically stimulated at $10 \mathrm{~Hz}$ for $10 \mathrm{sec}$ small processes often appeared nearly at the middle point of the postsynaptic membrane and then deeply invaginated into the presynaptic cytoplasm wearing the presynaptic membrane. The postsynaptic membrane composing this process was not associated with PSD (Fig. 1b). This small process breaking PSD resembled in morphology the "spinule" ever observed on the dendritic spine in CNS. ${ }^{1)}$ Referring to the findings of the preceding investigators in brain, we called this small process "synaptic spinule" in this article.

The synaptic spinule was small in size, $0.1-0.2 \mu \mathrm{m}$ in width and $0.3-0.5 \mu \mathrm{m}$ in height. The spinule subdivided PSD into two areas, and produced a perforated synapse having two presynaptic active zones standing opposite to the subdivided PSD (Fig. 1b). When the tetanic stimulation was prolonged, the spinules increased in population, and became bigger in size, more than $0.4 \mu \mathrm{m}$ in width. The top of growing spinules had a tendency to bend perpendicularly and then extend along the long axis of the preganglionic axon in this en-passant type synapse of the cat SCG.

Some postsynaptic dendrites have shown to form more than two spinules in one synapse (multiple type of spinules) after the stimulation of 1 min (Fig. 2). In such a case a single synapse was subdivided into two to five compartments, most of which had profiles of the active synapse with PSD and presynaptic active zones associated with synaptic vesicles. The shafts of the

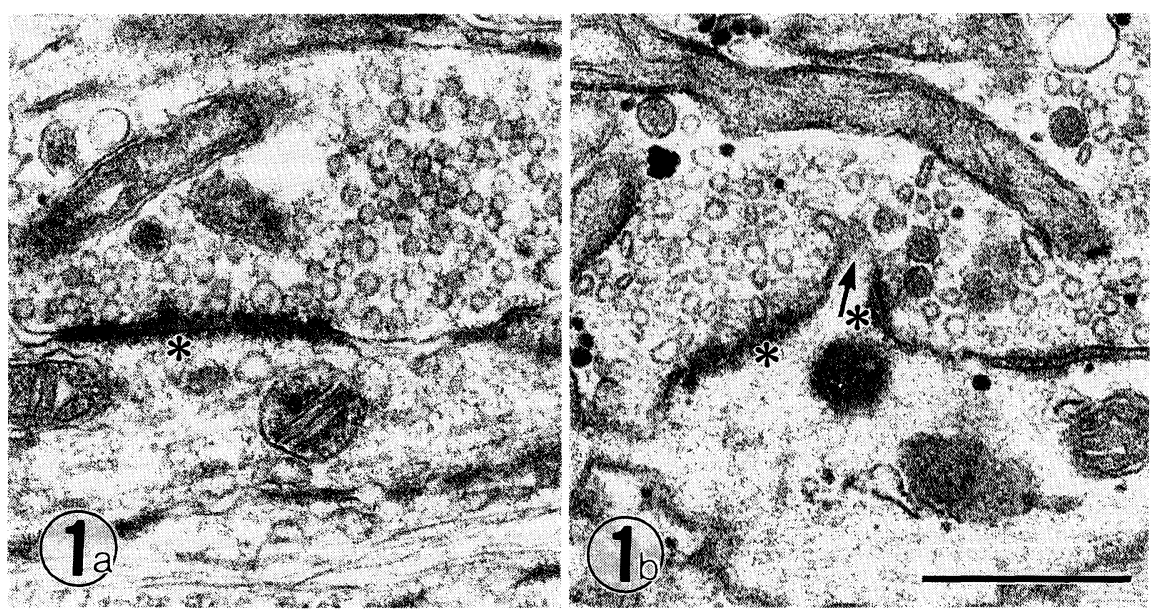

Fig. 1. An axodendritic synapse of cat SCG. (a) Unstimulated. The pre- and postsynaptic membranes were straight and parallel. The postsynaptic density (PSI)) (asterisk) continuously lined the postsynaptic membrane. (b) Stimulated at $10 \mathrm{~Hz}$ for $10 \mathrm{sec}$. A synaptic spinule (arrow) has been formed nearly at the middle point of PSI), producing a perforated synapse (asterisks). The spinule invaginated into the presynaptic terminal and subdivided a nerve terminal into two compartments. A cluster of synaptic vesicles and an active zone facing the subdivided PsI) across the synaptic cleft, were observed at each compartment. Bar equals 0.5, ॥m. 


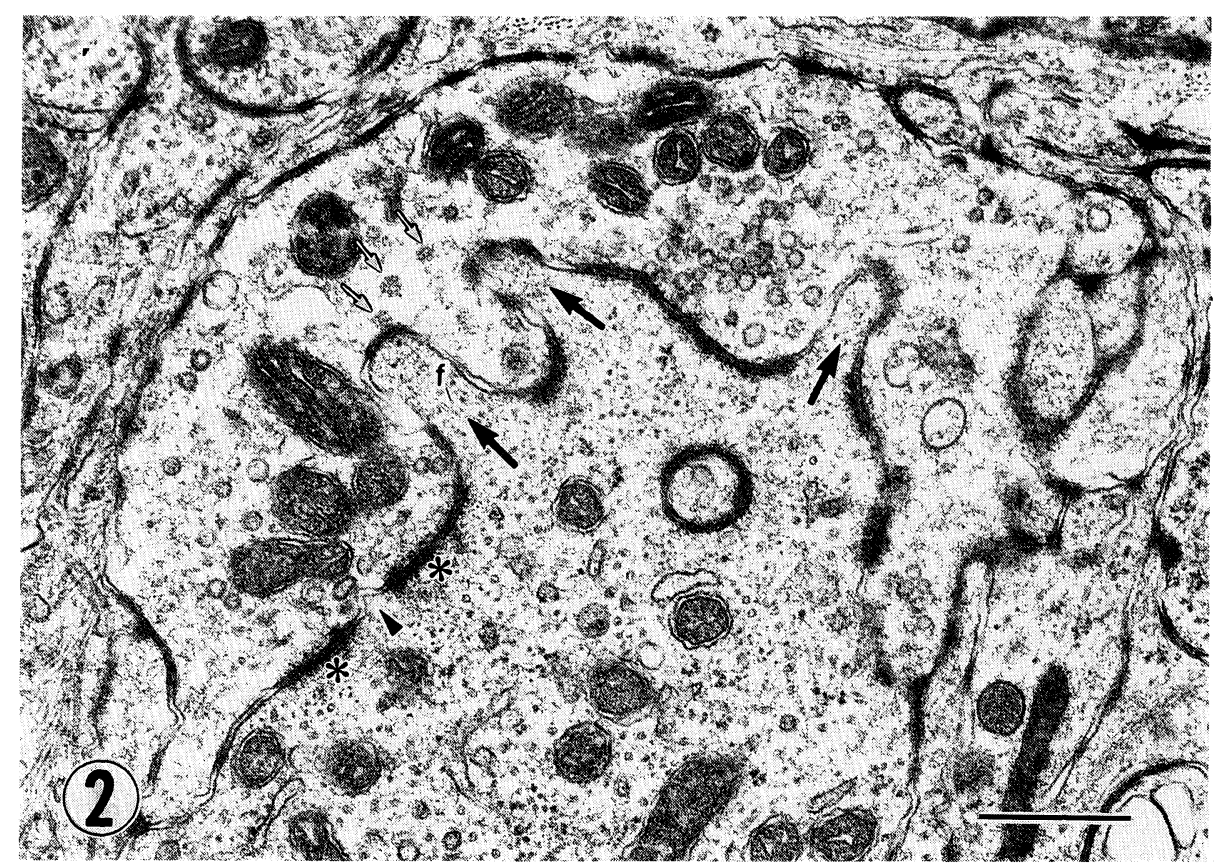

Fig. 2. An axodendritic synapse of SCG. Stimulated at $10 \mathrm{~Hz}$ for $1 \mathrm{~min}$. Multiple spinules (arrows) have been formed on a single postsynaptic dendrite. The presynaptic terminal had a few synaptic compartments. Coated resicles (open arrows) were found in one of the compartments. A small spinule (arrowhead) was observerl at a break of PSI) (asterisk). A few microfilaments (f) were found in the cytoplasm of spinules. HRP (horseradish peroxidase) was extracellularly applied before starting the stimulation. Bar equals $0.5 \mathrm{~mm}$.

growing spinules were often cut transversely within the cytoplasm of the nerve terminal in an electron micrograph, being shown as circular or ellipsoidal profiles enclosed with double membranes.

Some coated pits or vesicles were often found in the presynaptic cytoplasm around the spinule (Fig. 2). The cytoplasm of the spinule was continuous with that of the postsynaptic dendrite and seemed to be dense. This increase of the cytoplasmic density may relate to the appearance of microfilaments in the spinule.

Discussion. The present experiments examined the formation of a small process of the postsynaptic dendritic membrane in the cat SCG in response to a tetanic stimulation under normal blood supply. The small process, termed as "synaptic spinule" in the present report, stood out from the dendritic surface membrane concurrently with the appearance of PTP. The spinule formed a perforated synapse by producing a break in PSD nearly at the middle point (single type of spinule) and subdivided a synapse into two compartments by invaginating together with the presynatic membrane into the presynaptic cytoplasm at an earlier phase of PTP. When the tetani were prolonged, a few spinules were formed (multiple type of spinule) and subdivided the presynaptic cytoplasm into more than two compartments.

A finger-like extension from the dendritic spine, $0.1-0.2 \mu \mathrm{m}$ in width and $0.3-0.5 \mu \mathrm{m}$ in height, has been observed by the preceding investigators in the rat CNS. 1,2) This small protrusion has been named "spinule" and found to be formed on the dendritic spine under the activated states of the synapses such as LTP and kindling.:3),11) The preceding workers have suggested that the spinule formation results in the production of the perforated synapse. ${ }^{4), 10)}$ Similar synaptic changes were also found in a fish retina after the light adaptation. ${ }^{5), 11)}$ The generation of the perforated synapses have been presumed to produce an increase of the number of the synaptic active sites. ${ }^{10)}$ In addition, it has been suggested that the synaptic plasticity could involve a cycle between single and perforated synapses and the increase of the latter may correlate with a rise of the synatic efficacy. ${ }^{4)}$ Recently, Edwards ${ }^{(i)}$ has presented a structural model of LTP, where additional glutamate receptor molecules may accumulate at the PSD area following the influx of $\mathrm{Ca}^{2+}$ into the postsynaptic dendrite when the postsynaptic membrane is depolarized.

The synapse between the preganglionic nerve terminal and the ganglion cell of the cat SCG is a 
typical axodendritic and asymmetrical one. ${ }^{9)}$ The dendrite of the ganglionic cells in the unstimulated control material does not have any projections like the dendritic spines which are ordinarily and widely observed in the neurons in CNS. When the tetanic stimulation was given to the preganglionic nerve fibers of SCG, the synaptic spinule was found to stand out directly from the postsynaptic membrane with a straightly extended contour coincidentally with the appearance of PTP. One synaptic spinule was generally formed at each synapse (single type of spinule) at the earlier phase of PTP after 10 sec of stimulation. More spinules (multiple type of spinule) were generated in a single synapse at the late phase of PTP after 1 min of activation. The formation of the synaptic spinule resulted in a break of PSD, generating a perforated synapse. The spinule often invaginated into the presynaptic cytoplasm and subdivided a synapse into a few compartments, most of which had the active synaptic sites in morphology.

From the present observations and the preceding findings, it has been suggested that the synaptic spinule in the cat SCG agrees in many respects with the spinule found in the dendritic spine of the rat CNS or in the fish retina; location on the postsynaptic dendritic membrane, a finger-like shape, size, production of the perforated synapse as well as the compartmented synapse, and formation in response to the synaptic activation such as PTP or LTP. It is suggested that the synaptic spinule in the cat SCG is a morphological representation of the synaptic plasticity electrophysiologically shown as PTP, and that the spinule formation developing in response to the synaptic activation is one of the common mechanisms affecting the synaptic efficacy both in PNS and CNS.

\section{References}

1) Westrum, L. E., and Blackstad, T. W. (1962) J. Comp. Neurol. 119, 281-309.

2) Tarrant, S. B., and Routtenberg, A. (1977) Tissue Cell 9, 461-473.

3) Geinisman, Y., Morrell, F., and de Toledo-Morrell, L. (1990) Brain Res. 507, 325-331.

4) Geinisman, Y., de Toledo-Morrell, L., Morrell, F., Persina, I. S., and Rossi, M. (1993) Hippocampus 2, 445-456.

5) Wagner, H.-J., and Djamgoz, M. B. (1993) TINS 16, 201-206.

6) Edwards, F. A. (1995) TINS 18, 250-255.

7) Kadota, T., Mizote, M., Hori, M., Fujita, M., and Kadota, K. (1992) J. Electron Microsc. 41, 37-45.

8) Kadota, T., and Kadota, K. (1982) J. Electron Microsc. 31, $73-80$.

9) Elfvin, L-G. (1963) J. Ultrastruct. Res. 8, 441-476.

10) Lisman, J. E., and Harris, K. M. (1993) TINS 16, 141-147.

11) Harris, K. M., Jensen, F. E., and Tsao, B. (1992) J. Neurosci. 12, 2685-2705. 